\title{
Crianças e adolescentes residentes em zonas rurais apresentam maior prevalência de
}

\section{cárie?}

\author{
Do children and adolescents living in rural areas have a higher prevalence of caries? \\ ¿Los niños y adolescentes que viven en zonas rurales tienen una mayor prevalencia de caries?
}

Recebido: 11/12/2021 | Revisado: 18/12/2021 | Aceito: 19/12/2021 | Publicado: 03/01/2022

\author{
Aurélio de Oliveira Rocha \\ ORCID: https://orcid.org/0000-0002-9308-2118 \\ Universidade Federal de Santa Catarina, Brasil \\ E-mail: aureliorocha2015@gmail.com \\ Lucas Menezes dos Anjos \\ ORCID: https://orcid.org/0000-0001-5100-0789 \\ Universidade Federal de Santa Catarina, Brasil \\ E-mail: luks_anjos@hotmail.com \\ Thaine Oliveira Lima \\ ORCID: https://orcid.org/0000-0001-5220-9947 \\ Universidade de São Paulo, Brasil \\ E-mail: thaineo195@gmail.com \\ Pablo Silveira Santos \\ ORCID: https://orcid.org/0000-0001-9310-5642 \\ Universidade Federal de Santa Catarina, Brasil \\ E-mail: pablossant@gmail.com
}

\begin{abstract}
Resumo
O local de residência tem o potencial de moldar o comportamento e o estilo de vida dos indivíduos, podendo influenciar diretamente nos hábitos diários e causar patologias como a cárie dentária. O objetivo do presente estudo foi verificar se haveria disparidades entre a prevalência da doença cárie entre crianças e adolescentes residentes na zona rural e urbana. Foi realizada uma busca completa em estudos publicados nos últimos cinco anos nas bases de dados PubMed, LILACS, Science Direct e SciELO, utilizando os descritores "odontologia”, "zona rural”, "saúde bucal”, "criança” e "cárie”, inseridos nas plataformas de maneira cruzada utilizando a expressão booleana AND. Dentre os 209 artigos identificados, 200 foram excluídos por não se enquadrarem aos critérios pré-determinados. Portanto, nove artigos foram qualificados para inclusão e análise. Dentre os estudos analisados, 55,55\% apontam maior prevalência de cárie em residentes da zona rural, 22,22\% apontam maior prevalência nos residentes da zona urbana e 22,22\% apontam uma mínima diferença entre os grupos. Os residentes rurais estão mais propensos e apontam maiores índices de cárie dentária em relação aos residentes urbanos, os fatores ambientais podem interferir diretamente no aparecimento e fortalecimento dessa condição.
\end{abstract}

Palavras-chave: Odontologia; Zona rural; Saúde bucal; Criança; Cárie.

\begin{abstract}
The place of residence has the potential to shape the behavior and lifestyle of individuals, which can directly influence daily habits and cause pathologies such as tooth decay. The aim of the present study was to verify whether there would be disparities between the prevalence of caries disease among children and adolescents living in rural and urban areas. A complete search was carried out in studies published in the last five years in the PubMed, LILACS, Science Direct and SciELO databases, using the descriptors "dentistry", "rural area", "oral health", "child" and "cavities", inserted into the platforms crosswise using the boolean AND expression. Among the 209 articles identified, 200 were excluded for not meeting the predetermined criteria. Therefore, nine articles were eligible for inclusion and analysis. Among the studies analyzed, $55.55 \%$ indicate a higher prevalence of caries in rural residents, $22.22 \%$ indicate a higher prevalence in urban residents and $22.22 \%$ indicate a minimal difference between the groups. Rural residents are more likely and show higher rates of dental caries compared to urban residents, environmental factors can directly interfere in the appearance and strengthening of this condition.
\end{abstract}

Keywords: Dentistry; Rural area; Oral health; Child; Caries.

\section{Resumen}

El lugar de residencia tiene el potencial de moldear el comportamiento y el estilo de vida de las personas, lo que puede influir directamente en los hábitos diarios y causar patologías como la caries dental. El objetivo del presente estudio fue verificar si habría disparidades entre la prevalencia de la enfermedad de caries entre los niños y adolescentes que viven en áreas rurales y urbanas. Se realizó una búsqueda completa en los estudios publicados en los últimos cinco años en las 
Research, Society and Development, v. 11, n. 1, e10611124634, 2022

(CC BY 4.0) | ISSN 2525-3409 | DOI: http://dx.doi.org/10.33448/rsd-v11i1.24634

bases de datos PubMed, LILACS, Science Direct y SciELO, utilizando los descriptores "odontología", "zona rural", "salud bucal", "niño" y "caries", insertado en las plataformas de forma transversal mediante la expresión booleana AND. De los 209 artículos identificados, 200 fueron excluidos por no cumplir con los criterios predeterminados. Por lo tanto, nueve artículos fueron elegibles para su inclusión y análisis. Entre los estudios analizados, el 55,55\% indica una mayor prevalencia de caries en los residentes rurales, el 22,22\% indica una mayor prevalencia en los residentes urbanos y el $22,22 \%$ indica una mínima diferencia entre los grupos. Los residentes rurales son más propensos y muestran tasas más altas de caries que los residentes urbanos; los factores ambientales pueden interferir directamente con la apariencia y el fortalecimiento de esta condición.

Palabras clave: Odontología; Zona rural; Salud bucal; Niño; Caries.

\section{Introdução}

A saúde bucal caracteriza-se pelo equilíbrio da cavidade oral sem doenças ativas ou desconforto, contribuindo para a saúde geral do organismo; permitindo a fala, alimentação e comunicação efetiva. As crianças, diferentes dos adultos, necessitam de orientação e assistência para realização de cuidados e manutenção da saúde bucal (Li et al., 2015).

A presença de patologias na cavidade bucal pode afetar a saúde do indivíduo, causar comprometimento funcional e/ou estético e impactar sua qualidade de vida (Peres et al., 2019). Dentre diversas patologias, destaca-se a cárie dentária. Apesar de um aparente declínio na prevalência e progressão da cárie dentária nas últimas décadas, esta continua sendo um desafio de saúde pública mundial, pois é ainda considerada a doença crônica mais frequente da cavidade oral segundo a Organização Mundial da Saúde (OMS) (Peres et al., 2019). Embora a cárie dentária tenha diminuído, uma pesquisa nacional nos Estados Unidos entre 2015 e 2016 mostrou que aproximadamente $45.8 \%$ dos jovens de 2 a 19 anos tiveram ou tem cárie dentária em seus dentes decíduos e/ou permanentes (Fleming e Afful, 2018).

Um dos fatores modificadores da cárie dentária compreende a localização de moradia, referindo-se às áreas geográficas, ou seja, domicílios rurais e urbanos (Ardenghi et al., 2013). O local de residência tem potencial de moldar o comportamento e o estilo de vida dos indivíduos, podendo influenciar nos hábitos diários e no acesso aos serviços de saúde, inclusive ao tratamento odontológico (Ardenghi et al., 2013; Lešić et al., 2019). Quanto aos potenciais riscos vinculados à zona rural, pode-se destacar a ausência de fluoretação das águas e, em muitos casos, a baixa escolaridade dos responsáveis, além do difícil acesso ao atendimento odontológico (Freire et al., 2013). Em contrapartida, nas áreas urbanas podemos destacar o amplo acesso aos produtos industrializados ricos em carboidratos e açúcares, fator determinante para a instalação da cárie dentária (Freire et al., 2013; Tellez et al., 2006; Gushi et al., 2005).

A revisão integrativa compreende uma ampla abordagem metodológica, permitindo a inclusão de estudos experimentais e não-experimentais para uma compreensão completa da questão analisada (Whittemore e Knafl, 2005). Incorpora uma gama de propósitos: definição de conceitos, revisão de teorias e evidências e análise de problemas metodológicos de um tópico particular. A ampla amostra, em conjunto com a multiplicidade de propostas, deve gerar um panorama consistente e compreensível de conceitos complexos, teorias ou problemas de saúde frequentes como a cárie dentária (Whittemore e Knafl, 2005).

O ambiente costuma moldar o comportamento dos indivíduos, sendo capaz de refletir diretamente em sua saúde (Lešić et al., 2019). Analisar os artigos que abordam as áreas mais prevalentes em cárie na população infantil contribui diretamente para as ciências da saúde, influenciando a pesquisa e orientando as políticas públicas de saúde, contribuindo assim para a redução de uma das doenças crônicas mais frequente em crianças e adolescentes em todo o mundo. Assim, o objetivo do presente estudo é avaliar as disparidades entre a prevalência da doença cárie entre crianças e adolescentes residentes na zona rural e urbana.

\section{Metodologia}

O presente estudo configura-se como uma revisão bibliográfica integrativa, método específico de pesquisa que proporciona a síntese do conhecimento e a avaliação crítica de resultados de estudos significativos e evidências sobre determinado assunto (Whittemore e Knafl, 2005). Dessa forma, permite que seu resultado mostre a relevância e o estado atual do conhecimento 
acerca do tema investigado, além de nortear sobre a real situação da temática. Ademais, exibe-se os pontos fortes e fracos que necessitarão de uma maior atenção e aprimoramento das práticas e políticas públicas de saúde (Souza et al., 2010).

Inicialmente, foi elaborada a seguinte pergunta norteadora: "Crianças e adolescentes residentes em zonais rurais possuem maior prevalência de cárie dentária, em comparação com crianças e adolescentes residentes em zonas urbanas?” Estratégias de buscas foram construídas para as seguintes bases de dados eletrônicas: Medical Literature Analysis and Retrieval System Online (Medline/PubMed), Literatura Latino-Americana e do Caribe em Ciências da Saúde (LILACS), Science Direct e Scientific Electronic Library Online (SciELO). Foram utilizadas as combinações dos descritores: "odontologia", "zona rural", "saúde bucal", "criança" and "cárie", todos os termos cadastrados nos Descritores em Ciências da Saúde (DeCS), juntamente com o operador booleano "AND". Assim, houve a consideração de artigos publicados nos últimos cinco anos, ou seja, no período entre julho de 2016 a julho de 2021, a fim de agrupar as pesquisas com informações mais recentes.

A seleção inicial dos artigos ocorreu mediante os critérios de elegibilidade. Foram incluídos artigos em qualquer idioma, com texto completo disponível e que abordavam a condição de saúde e cárie dentária em crianças e adolescentes de comunidades rurais e urbanas. Após a pesquisa e seleção segundo os critérios descritos, foi realizada a leitura do título e resumo, a fim de determinar os estudos que realmente se enquadravam na temática pesquisada e que seriam relevantes para a pesquisa. Houve a exclusão de estudos em adultos, revisões de literatura, estudos que não contemplassem a temática específica e artigos sem texto completo disponível.

\section{Resultados}

A combinação dos descritores nas bases de dados resultou em 209 artigos, destes 200 foram excluídos por não apresentarem texto completo disponível, publicação com menos de cinco anos e/ou não tratar do assunto proposto, ou seja, por não preencherem os critérios de inclusão pré-definidos, assim 09 artigos foram selecionados para leitura completa e extração dos dados analisados. A descrição detalhada da etapa de seleção pode ser observada na Figura1.

Figura 1 - Fluxograma de seleção dos estudos, apresenta-se número de artigos de cada base e seleção dos mesmos. 

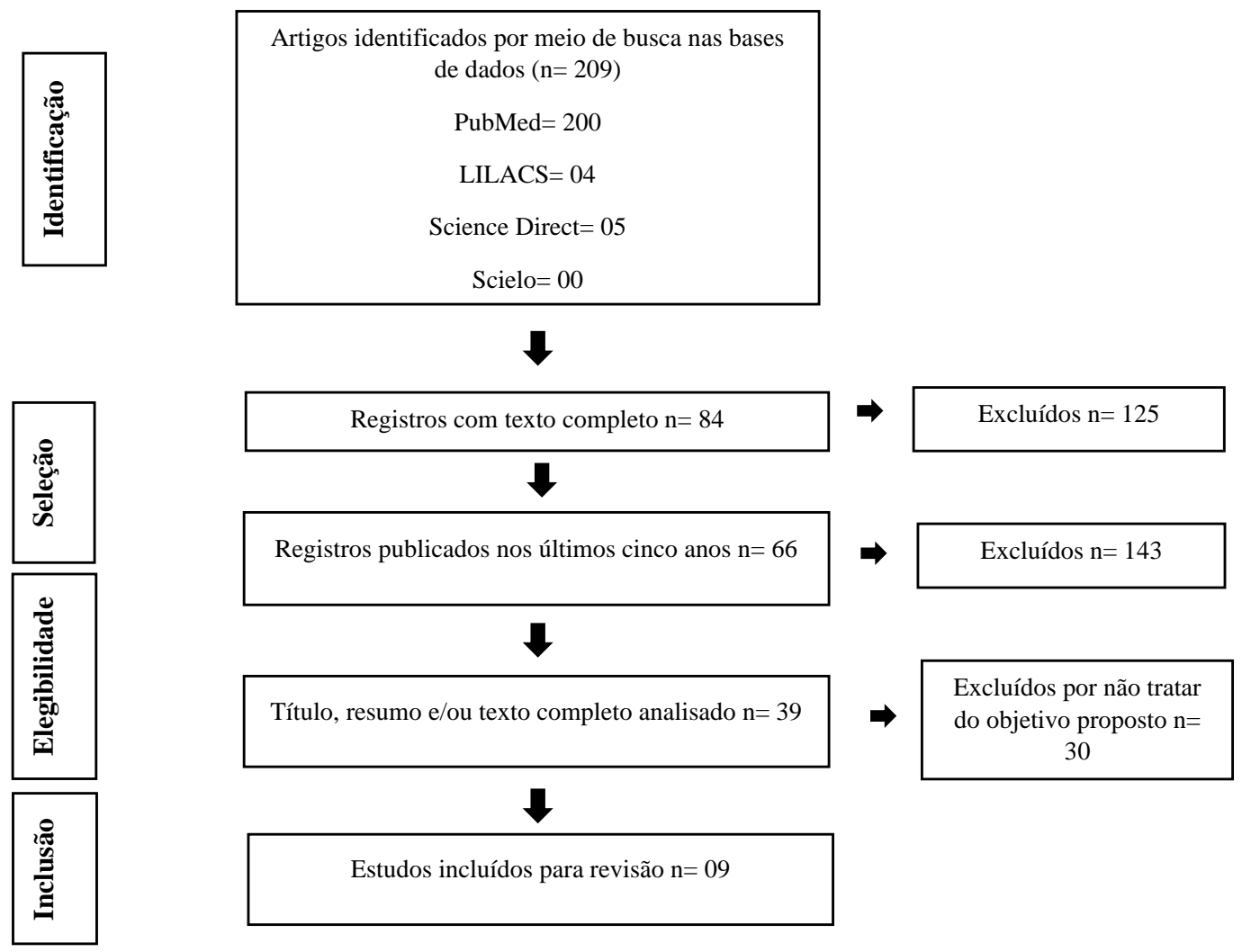

Fonte: Rocha AO, et al. (2021).

Os artigos selecionados para realização desse estudo e dados extraídos para análise estão descritos no Quadro 1.

Quadro 1: Dados extraídos dos artigos analisados, todos utilizaram o índice CPO-D/ceo-d.

\begin{tabular}{|c|c|c|c|}
\hline $\begin{array}{c}\text { Autores } \\
\text { Ano e local de publicação }\end{array}$ & $\begin{array}{c}\text { Amostra Total (T) } \\
\text { Rural (R) } \\
\text { Urbana (U) }\end{array}$ & Idade & Principais resultados \\
\hline $\begin{array}{l}\text { Nora AD et al. } \\
2020 \\
\text { Brasil }\end{array}$ & $\begin{array}{l}\mathrm{T}=373 \\
\mathrm{R}=122 \\
\mathrm{U}=251\end{array}$ & $9-14$ & $\begin{array}{l}\text { Escolares urbanos tiveram } 57 \% \text { mais chances de apresentar cárie } \\
\text { que os rurais. }\end{array}$ \\
\hline $\begin{array}{l}\text { Giacaman RA et al. } \\
2018 \\
\text { Chile }\end{array}$ & $\begin{array}{l}\mathrm{T}=552 \\
\mathrm{~T}=486\end{array}$ & $\begin{array}{l}12 \\
15\end{array}$ & $\begin{array}{l}\text { Crianças de } 12 \text { anos da área rural apresentaram prevalência de cárie } \\
\text { de } 67,50 \% \text {, e da área urbana } 54,04 \% \text {. Adolescentes de } 15 \text { anos, nas } \\
\text { áreas rurais } 73,58 \% \text { e urbanas } 64,59 \% \text {. }\end{array}$ \\
\hline $\begin{array}{l}\text { Chinna SK et al. } \\
2019 \\
\text { Índia }\end{array}$ & $\begin{array}{l}T=1240 \\
R=620 \\
U=620\end{array}$ & 12 & $\begin{array}{l}\text { A prevalência de cárie em áreas urbanas e rurais em escolares foi } \\
\text { de } 63,5 \% \text { e } 64,5 \% \text {, respectivamente. }\end{array}$ \\
\hline $\begin{array}{l}\text { Llano-Pérula MC et al. } \\
\qquad \begin{array}{l}2020 \\
\text { Peru }\end{array}\end{array}$ & $\mathrm{T}=1.062$ & 14 & $\begin{array}{l}\text { A prevalência de cárie foi maior em Cuzco }(97,65 \%) \text {, seguida de } \\
\text { Titicaca }(88,81 \%) \text { e Lima }(76,42 \%) \text {. }\end{array}$ \\
\hline $\begin{array}{l}\text { Singh BT et al. } \\
2020 \\
\text { Índia }\end{array}$ & $\mathrm{T}=1000$ & $3-16$ & $\begin{array}{l}\text { A prevalência de cárie em dentes permanentes foi maior na zona } \\
\text { rural com um valor médio de } 0,51 \text { em comparação com a } \\
\text { população urbana. }\end{array}$ \\
\hline $\begin{array}{l}\text { Qin D et al. } \\
2019\end{array}$ & $\begin{array}{l}\mathrm{T}=5057 \\
\mathrm{R}=2549\end{array}$ & $10-12$ & $\begin{array}{l}\text { A prevalência de cárie nas áreas rurais }(46,6 \%) \text { é maior que nas } \\
\text { cidades }(31,7 \%) \text {. }\end{array}$ \\
\hline
\end{tabular}


Research, Society and Development, v. 11, n. 1, e10611124634, 2022

(CC BY 4.0) | ISSN 2525-3409 | DOI: http://dx.doi.org/10.33448/rsd-v11i1.24634

\begin{tabular}{|c|c|c|c|}
\hline China & $\mathrm{U}=2508$ & & \\
\hline $\begin{array}{c}\text { Lešić S et al. } \\
2019 \\
\text { Croácia }\end{array}$ & $\begin{array}{c}T=1589 \\
R=265 \\
U=1324\end{array}$ & $6-12$ & $\begin{array}{l}50,0 \% \text { das crianças apresentavam cárie, sendo } 46,0 \% \text { delas } \\
\text { residentes da zona urbana e } 70,2 \% \text { da zona rural. }\end{array}$ \\
\hline $\begin{array}{c}\text { Kamińska A et al. } \\
2016 \\
\text { Polônia }\end{array}$ & $\mathrm{T}=844$ & $3-6$ & $\begin{array}{l}\text { A incidência de cárie foi de } 52,61 \% \text {, com valor médio de } 4,31 \text {; } \\
\text { crianças da zona urbana essa relação foi de } 4,15 \text { e no campo } 4,7 \text {. }\end{array}$ \\
\hline $\begin{array}{l}\text { Al-Rafee MA et al. } \\
\qquad 2019 \\
\text { Arábia Saudita }\end{array}$ & $\mathrm{T}=1986$ & 6-15 & $\begin{array}{l}\text { Tanto a taxa de cárie quanto a gravidade foram maiores nas áreas } \\
\text { rurais que nas urbanas }\end{array}$ \\
\hline
\end{tabular}

Fonte: Rocha AO, et al. (2021).

Os trabalhos selecionados foram desenvolvidos recentemente, concentrando publicações nos últimos cinco anos (julho 2016 - julho 2021). Dois estudos foram realizados na Índia (Chinna et al., 2019; Singh et al., 2020), um no Brasil (Dalla Nora et al., 2020), um no Chile (Giacaman et al., 2018), um no Peru (Cadenas de Llano-Pérula et al., 2020), um na China (Qin et al., 2019), um na Croácia (Lešić et al., 2019), um na Polônia (Kamińska et al., 2016) e um na Arábia Saudita (Al-Rafee et al., 2019). Sendo assim, $77,77 \%$ das pesquisas foram realizadas em países subdesenvolvidos ou em desenvolvimento e 22,22\% em países desenvolvidos.

A amostra foi composta por crianças e adolescentes residentes na zona rural ou urbana, todos os trabalhos (100\%) utilizaram os índices preconizado pela Organização Mundial da Saúde para dentes cariados, perdidos e obturados (CPO-D) para dentição permanente e cariados, indicados para extração e obturados (ceo-d) para dentição decídua. Dentre os estudos analisados, 55,55\% apontam maior prevalência de cárie em residentes da zona rural, 22,22\% apontam maior prevalência nos residentes da zona urbana e 22,22\% apontam uma mínima diferença entre os grupos.

\section{Discussão}

Este estudo foi realizado com o intuito de avaliar as disparidades entre a prevalência da doença cárie entre crianças e adolescentes residentes na zona rural e urbana em diversos países. A identificação de fatores causais e focos epidemiológicos vinculados a essa patologia é crucial para realizar uma intervenção efetiva.

A cárie dentária é uma doença crônica que gera desconforto e afeta a qualidade de vida das crianças e adolescentes, devido sua alta prevalência e severidade, necessita de uma maior atenção por parte de pesquisadores e clínicos, principalmente em grupos coletivos, como residentes da zona rural e urbana (Tuon et al., 2007; Al-Rafee et al., 2019). De acordo com os estudos avaliados, foi possível observar alto índice dessa condição entre a população infantil.

A cárie dentária é uma doença multifatorial que apresenta considerável declínio, principalmente em países desenvolvidos (Al-Akwa e Al-Maweri, 2018). Por outro lado, em países subdesenvolvidos, a baixa condição socioeconômica da população pode ser uma condição agravante fundamental para o aumento da prevalência da doença cárie (Al-Akwa e Al-Maweri, 2018; Kuriakose et al., 2015; Cortelli et al., 2004). Dentre os países vinculados aos resultados do presente estudo, sete deles configuram países subdesenvolvidos ou em desenvolvimento que exibem alta prevalência de cárie na dentição infantil, entretanto a Croácia e Polônia - países desenvolvidos - ainda exibem altas taxas de cárie em crianças e adolescentes da zona rural quando comparadas as demais nacionalidades. 
O índice CPOD apresenta o seguinte significado: CPO compreende-se nas palavras "cariados", "perdidos” e "obturados", e o D indica que o dente é a unidade de medida em questão, formulado por Klein e Palmer, em 1937, é usado pela Organização Mundial de Saúde (OMS) para avaliar a prevalência da cárie dentária em diversos países, amplamente utilizado em diversas pesquisas devido sua alta confiabilidade e interpretação simplificada (Cortelli et al., 2004). O índice ceo-d corresponde ao CPOD vinculado à dentição decídua, mas inclui só os dentes cariados (c), com extração indicada (e) e obturados (o), não apresenta os extraídos pois é dificil saber se a perda foi por cárie ou esfoliação fisiológica. Todos os estudos avaliados utilizaram o CPOD/ceod como índice para a avaliação da prevalência de cárie (Kamińska et al., 2016).

Dois estudos (Dalla Nora et al., 2020; Cadenas de Llano-Pérula et al., 2020) apontaram crianças e adolescentes residentes da zona urbana como a população mais propensa a apresentar atividade de cárie, fator que pode estar relacionado aos hábitos alimentares e comportamentais. Residentes da zona urbana apresentam um maior acesso a dieta cariogênica, ou seja, produtos ricos em açúcares altamente associados ao início da lesão cariosa seja em casa ou mesmo na escola (Fleming e Afful, 2018).

Residentes da zona rural não apresentam amplo acesso aos centros comerciais, supermercados e lanchonetes, o que restringe assim o contato com produtos industrializados, potencialmente cariogênicos (Freire et al., 2013). Por outro lado, o acesso ao atendimento odontológico, educação sobre saúde bucal e produtos fluoretados apresenta uma grande limitação para essa população. Com isso, torna-se propício um alto risco de desenvolver a cárie, condições estas que contribuiram fortemente para termos assim encontrados cinco estudos indicando uma maior prevalência de cárie em crianças residentes da zona ruraL (Lešić et al., 2019; Chinna et al., 2019; Giacaman et al., 2018; Qin et al., 2019; Al-Rafee et al., 2019).

Estudos relatam uma forte relação entre condições bucais e fatores sociodemográficos como idade, educação, sexo, etnia e renda familiar (Correa-Faria et al., 2013; de Oliveira et al., 2013; Moimaz et al., 2014). Crianças vinculadas à famílias de baixa renda são mais propensas a sofrer de cárie dentária que aquelas economicamente favorecidas (de Oliveira et al., 2013). Outro ponto importante firmado na literatura é sobre a escolaridade dos pais, crianças com responsáveis detentores de alta escolaridade apresentam menos risco de cárie que filhos de pais com baixa escolaridade (Moimaz et al., 2014). A conscientização de pais e responsáveis sobre a prevenção da cárie é de suma importância para paralisação e aparecimento de novas lesões cariosas em crianças e adolescentes residentes em qualquer zona.

Independente do ambiente em que se vive, crianças e adolescentes apresentam vínculos com fatores predisponentes e determinantes da cárie dental, o que explicaria a ausência de diferença significativa entre esses grupos em dois estudos (Singh et al., 2020; Kamińska et al., 2016). Dessa forma, todos os indivíduos desta faixa etária necessitam de uma atenção especial voltada para suas limitações e intervenções efetivas que contribuam para a redução das altas taxas de prevalência dessa patologia.

\section{Conclusão}

Os residentes rurais estão mais propensos e apontam maiores índices de cárie dentária em relação aos residentes urbanos. Fatores ambientais podem interferir diretamente no aparecimento e fortalecimento dessa condição. A identificação da cárie dentária entre as dentições decídua e permanente em crianças e adolescentes ainda exibe taxas expressivas de prevalência, observou-se a necessidade de revisões sistemáticas com maiores detalhes do tema abordado.

\section{Referências}

Al-Akwa, A. A., \& Al-Maweri, S. A. (2018). Dental caries prevalence and its association with fluoride level in drinking water in Sana'a, Yemen. European journal of dentistry, 12(01), 015-020.

Al-Rafee, M. A., AlShammery, A. R., AlRumikan, A. S., \& Pani, S. C. (2019). A comparison of dental caries in urban and rural children of the Riyadh region of Saudi Arabia. Frontiers in public health, 7, 195.

Ardenghi, T. M., Piovesan, C., \& Antunes, J. L. F. (2013). Inequalities in untreated dental caries prevalence in preschool children in Brazil. Revista de saude publica, 47, 129-137. 
Research, Society and Development, v. 11, n. 1, e10611124634, 2022 (CC BY 4.0) | ISSN 2525-3409 | DOI: http://dx.doi.org/10.33448/rsd-v11i1.24634

Cadenas de Llano-Pérula, M., Ricse, E., Fieuws, S., Willems, G., \& Orellana-Valvekens, M. F. (2020). Malocclusion, dental caries and oral health-related quality of life: a comparison between adolescent school children in urban and rural regions in Peru. International journal of environmental research and public health, 17(6), 2038

Chinna, S. K., Acharya, A. K., \& Chinna, R. (2019). Oral health status and treatment needs of 12-year-old school children among urban and rural areas of Raichur Taluk, Karnataka, India. Indian journal of community medicine: official publication of Indian Association of Preventive \& Social Medicine, 44 (Suppl 1), S27.

Correa-Faria, P., Martins-Junior, P. A., Vieira-Andrade, R. G., Marques, L. S., \& Ramos-Jorge, M. L. (2013). Factors associated with the development of early childhood caries among Brazilian preschoolers. Brazilian oral research, 27, 356-362.

Cortelli, S. C., Cortelli, J. R., Prado, J. S., Aquino, D. R., \& Jorge, A. O. (2004). Fatores de risco a cárie e CPOD em crianças com idade escolar. Brazilian Dental Science, 7(2).

Dalla Nora, Â., Dalmolin, A., Gindri, L. D., Moreira, C. H. C., Alves, L. S., Zenkner, J. E. D. A. (2020). Oral health status of schoolchildren living in rural and urban areas in southern Brazil. Braz Oral Res., 34.

de Oliveira, T. C., da Silva, D. A., de Freitas, Y. N. L., da Silva, R. L., de Castro Pegado, C. P., \& de Lima, K. C. (2013). Socio-demographic factors and oral health conditions in the elderly: a population-based study. Archives of gerontology and geriatrics, 57(3), 389-397.

Fleming, E., \& Afful, J. (2018). Prevalence of total and untreated dental caries among youth: United States, $2015-2016$.

Freire, M. D. C. M., Reis, S. C. G. B., Figueiredo, N., Peres, K. G., Moreira, R. D. S., \& Antunes, J. L. F. (2013). Individual and contextual determinants of dental caries in Brazilian 12-year-olds in 2010. Revista de saude publica, 47, 40-49.

Giacaman, R. A., Bustos, I. P., Bazán, P., \& Mariño, R. J. (2018). Oral health disparities among adolescents from urban and rural communities of central Chile.

Gushi, L. L., Soares, M. D. C., Forni, T. I. B., Vieira, V., Wada, R. S., \& Sousa, M. D. L. R. D. (2005). Dental caries in 15-to-19-year-old adolescents in São Paulo State, Brazil, 2002. Cadernos de Saúde Pública, 21(5), 1383-1391.

Kaminska, A., Szalewski, L., Batkowska, J., Wallner, J., Wallner, E., Szabelska, A., \& Borowicz, J. (2016). The dependence of dental caries on oral hygiene habits in preschool children from urban and rural areas in Poland. Ann Agric Environ Med, 23(4), 660-665.

Kuriakose, S., Prasannan, M., Remya, K. C., Kurian, J., \& Sreejith, K. R. (2015). Prevalence of early childhood caries among preschool children in Trivandrum and its association with various risk factors. Contemporary clinical dentistry, 6(1), 69.

Lešić, S., Dukić, W., Kriste, Z. Š., Tomičić, V., \& Kadić, S. (2019). Caries prevalence among schoolchildren in urban and rural Croatia. Central European journal of public health, 27(3), 256-262.

Li, M.Y., Zhi, Q.H., Zhou, Y., Qiu, R.M., Lin, H.C. (2015). Impact of Early Childhood Caries on oral health-related quality of life of preschool children. Eur J Paediatr Dent. 16, 65-72.

Moimaz, S. A. S., Fadel, C. B., Lolli, L. F., Garbin, C. A. S., Garbin, A. J. Í., \& Saliba, N. A. (2014). Social aspects of dental caries in the context of mother-child pairs. Journal of Applied Oral Science, 22(1), 73-78.

Peres, M. A., Macpherson, L. M., Weyant, R. J., Daly, B., Venturelli, R., Mathur, M. R., ,.. \& Watt, R. G. (2019). Oral diseases: a global public health challenge. The Lancet, 394(10194), 249-260.

Singh, B. T., Pranitha, V., Dwijendra, K. S., Nagarjuna, G., Shaik, N., \& Kavya, R. (2020). Oral health status and treatment needs of children with special health care needs in rural and urban areas of Hyderabad-A cross sectional study. Journal of Family Medicine and Primary Care, 9(12), 6158.

Souza, M. T. D., Silva, M. D. D., \& Carvalho, R. D. (2010). Revisão integrativa: o que é e como fazer. Einstein (São Paulo), 8, 102-106.

Tellez, M., Sohn, W., Burt, B. A., \& Ismail, A. I. (2006). Assessment of the relationship between neighborhood characteristics and dental caries severity among low-income African-Americans: a multilevel approach. Journal of public health dentistry, 66(1), 30-36.

Tuon, A. C. L. F., de LACERDA, J. T., \& Traebert, J. (2007). Prevalência de cárie em escolares da zona rural de Jacinto Machado, SC, Brasil. Pesquisa Brasileira em Odontopediatria e Clínica Integrada, 7(3), 277-284.

Qin, D., Jiang, H. F., Shen, L., Zhang, C., Chai, Z. W., \& Wang, J. H. (2019). Prevalence of dental caries and associated factors among 10-12-year-old students in Chongqing. Hua xi kou qiang yi xue za zhi= Huaxi kouqiang yixue zazhi= West China journal of stomatology, 37(6), 608-614.

Whittemore, R., \& Knafl, K. (2005). The integrative review: updated methodology. Journal of advanced nursing, 52(5), 546-553. 